\title{
Acute Streptococcus pneumoniae lung infection: Mouse model and characterisation of the immune response.
}

Anne-Katrien Stark ( $\square$ aks82@cam.ac.uk)

Division of Immunology, Department of Pathology, University of Cambridge, Cambridge CB2 1QP, UK

Klaus Okkenhaug ( $\nabla$ ko256@cam.ac.uk)

Division of Immunology, Department of Pathology, University of Cambridge, Cambridge CB2 1QP, UK

Edward Banham-Hall

Cambridge University Hospitals NHS Trust, Hills Road, Cambridge, CB2 0QQ

\section{Method Article}

Keywords: Intranasal infection, Mouse model, Streptococcus pneumoniae, Flow cytometry

Posted Date: September 24th, 2018

DOI: https://doi.org/10.1038/protex.2018.114

License: (c) (1) This work is licensed under a Creative Commons Attribution 4.0 International License.

Read Full License 


\section{Abstract}

_Streptococcus pneumoniae_ is a globally important pathogen. We describe a mouse model of _S. pneumoniae_ respiratory tract infection through intranasal administration of bacteria which progress to bacteraemia and systemic disease. This model therefore allows assessment of different stages of infection and can be used to study local and systemic immune responses to vaccines and other treatment strategies.

\section{Introduction}

_S. pneumoniae_ $\backslash$ (pneumococcus) is a gram positive, extracellular bacterium. Although it exists as a commensal in the upper respiratory tract of roughly $10 \%$ of adult carriers, it has the potential to cause severe disease in susceptible individuals, most notably young children, the elderly and those with immunodeficiency. Pneumococcal disease results from local spread, aspiration or bacteraemia, and range from otitis media and sinusitis to pneumonia, meningitis and sepsis \[1]. Secondary _S. pneumoniae_ infections following influenza A infection are common, and_S. pneumoniae_also play a significant role in exacerbation of chronic lung pathologies such as COPD $\backslash[2,3]$. Two effective capsular polysaccharide vaccines are in widespread use. Pneumovax is a T-independent 23-valent vaccine, and Prevenar is a 13-valent protein conjugated T-dependent vaccine. However, vaccination protects only against serotypes included in the vaccine, and serotype replacement can mitigate efficacy at a population level over time \[4]. Mouse models of _S. pneumoniae_ infection and disease progression play an important role in increasing our understanding of host-pathogen interaction and identifying the factors that modulate_S. pneumoniae_ pathogenicity as well as evaluating universal protein or whole cell based vaccine strategies $\backslash[4]$. The properties of the mouse strain as well as_S. pneumoniae_strain and serotype should be carefully considered alongside the infection route and dose to ensure that the experimental design is appropriate to the research question $\backslash[5]$. In this protocol we describe the establishment of an intranasal_S. pneumoniae_lung infection model and characterisation of the resulting immune response which allows us to study different stages of disease progression including upper respiratory tract infection, pneumonia, bacteraemia as well as bacterial clearance and recovery. This model enabled us to assess the effects of genetic manipulation and/or treatment on disease progression and the immune response, and led to the discovery that B cells could exacerbate acute_S. pneumoniae_infections by an antibody independent mechanism $\backslash[6]$.

\section{Reagents}

**Animals** We used male and female transgenic mice between 8-15 weeks of age with age and sex matched wildtype controls in all experiments. All mice were bred on a C57BL/6J background. All experiments were performed in accordance with local standards and the Animals $\backslash$ (Scientific Procedures) Act 1986, licence PPL 70/7661 and were approved by the Babraham Institute Animal Welfare and Ethics Review Body. Mice were bred and maintained at the Babraham Institute Biological Support Unit. Regular health monitoring confirmed that stock and holding rooms were free of primary pathogens and additional 
agents listed in the FELASA recommendations \[7]. Ambient temperature and lighting was kept between $19-21^{\circ} \mathrm{C}$ with a relative humidity of $52 \%$ and a 12-hour light: 12-hour dark cycle including 15 min 'dawn' and 'dusk' periods of subdued lighting. Mice were housed in individually ventilated cages with 1-5 animals per cage and were fed CRM $\backslash(P)$ VP diet $\backslash$ (Special Diet Services) ad libitum. Animals received sunflower or millet seeds at the time of cage-cleaning as part of their environmental enrichment. **Anaesthesia** • Isoflurane: IsoFlo 100\% w/w Inhalation Vapour, liquid \(Zoetis) **S. pneumoniae stock**_Note: S. pneumoniae is a category 2 pathogen capable of causing disease in humans. Ensure that all work involving viable bacteria is completed in a containment level 2 laboratory and that appropriate risk assessments are in place._ We received_S. pneumoniae_TIGR4, serotype 4 as a kind gift from Professor Jeremy Brown, University College London. This strain is a clinical isolate and not genetically modified. Serotype 4 has been shown to be virulent in mice $\backslash[8]$ and is included in the two clinically available vaccines: Prevenar-13 and Pneumovax. A variety of other serotypes and strains are available from ATCC and BEl Resources. **Media** •_S. pneumoniae_ culture media: Add 36.4g ToddHewitt broth powder $\backslash($ Oxoid CM0189) and $5 \mathrm{~g}$ yeast extract $\backslash($ Oxoid LP0021) to $1 \mathrm{~L}$ sterile milliQ water, heat and mix until dissolved, then autoclave. B Blood-Agar plates: Prepare 500mL LB-Agar $\backslash($ ThermoFisher scientific 22700), autoclave and allow to cool to $50^{\circ} \mathrm{C}$ in a water-bath, then add $25 \mathrm{~mL}$ defibrinated sheep's blood \(Oxoid SR0051). Mix well, pour into sterile $90 \mathrm{~mm}$ petri dishes and allow to set under aseptic conditions. Add Optochin antibiotic disks $\backslash$ (Sigma-Aldrich 74042-50DISCS-F) to selected plates. Plates can be stored under aseptic conditions at $4^{\circ} \mathrm{C}$ for up to 5 days. Phosphate buffered saline $\backslash($ PBS): Low endotoxin Dulbeccos's phosphate buffered saline without Ca2+ or Mg2+ \(Sigma-Aldrich D8537). • Glycerol \(Sigma-Aldrich G5516) **Vaccines** Pneumovax II \(pneumococcal polysaccharide vaccine, Sanofi Pasteur MSD). One $0.5 \mathrm{~mL}$ dose contains $50 \mu \mathrm{g} / \mathrm{mL}$ of each serotype $\backslash(1-5,6 \mathrm{~B}, 7 \mathrm{~F}, 8,9 \mathrm{~N}, 9 \mathrm{~V}, 10 \mathrm{~A}$, 11A, 12F, 14, 15B. 17F, 18C, 19F, 19A, 20, 22F, 23F and 33F). Dilute one 0.5mL dose 1:12.5 in sterile PBS for administration to mice. Prevenar $13 \backslash$ (pneumococcal 13-valent conjugate vaccine, Diphteria CRM197 protein, Pfizer). One $0.5 \mathrm{~mL}$ dose contains $22 \mu \mathrm{g}$ of each serotype: $1-5,6 \mathrm{~A}, 7 \mathrm{~F}, 9 \mathrm{~V}, 14,18 \mathrm{C}, 19 \mathrm{~A}, 23 \mathrm{~F}$ polysaccharides and $4.4 \mu \mathrm{g}$ of serotype $6 \mathrm{~B}$ polysaccharide; $34 \mu \mathrm{g}$ CRM197 carrier protein, $100 \mu \mathrm{g}$ polysorbate $80,295 \mu \mathrm{g}$ succinate buffer and $125 \mu \mathrm{g}$ aluminium phosphate adjuvant. Dilute one $0.5 \mathrm{~mL}$ dose 1:6.4 in sterile PBS for administration to mice. ${ }^{\star *}$ Immune cell isolation ${ }^{\star \star} \cdot$ Red blood Cell lysis buffer: Red blood cell lysis buffer Hybri-Max \(Sigma-Aldrich R7757) • Mouse Lung dissociation kit \ (Miltenyi, 130-095-927) Prepared according to manufacturer's instructions. • Percoll \(Sigma-Aldrich P1644): Prepare isotonic Percoll by adding 1 part sterile 10x concentrated PBS to 9 parts Percoll. Then prepare a $37.5 \%$ working solution of isotonic Percoll in sterile 1x PBS. ${ }^{*}$ Flow cytometry* • Antibodies: see Table 1 See figure in Figures section. • Staining buffer: 0.5\% BSA in PBS. • Fixation buffer: $4 \%$ paraformaldehyde $\backslash$ (Biolegend 420801) - Transcription factor staining buffer set $\backslash$ (eBioscience 00-5523-

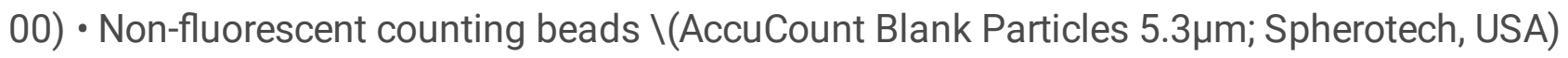

\section{Equipment}

**Anaesthesia** Vet-tech anaesthetic system, including recoil hoses and quick release connections • Isoflurane Key Fill Vapouriser CM \(Vet-Tech AN003A) • Isoflurane key fill applicator $\backslash($ AN003B) • 
Continuous Anaesthetic chamber $\backslash($ small, red $) \backslash($ vet-tech AN010SR) • Oxygen supply • Isoflurane scavenging system **Tissue dissociation for CFU counts** • Bullet blender $\backslash$ (Next Advance, standard model) $\cdot 3.2 \mathrm{~mm}$ Stainless steel beads $\backslash$ (Next Advance) Eppendorf $\backslash$ (Safelock) $2 \mathrm{~mL}$ microcentrifuge tubes $\backslash$ (Sigma-Aldrich) **Immune cell isolation** • GentleMACS tissue dissociator $\backslash($ Miltenyi) $\backslash($ Optional) • Flow cytometer: LSRFortessa see Table 2 for laser power and filter sets. See figure in Figures section. **General equipment** • Pipettes and tips: $\backslash($ p1000, p200, p20) \(Starlab) • Consumables: $50 \mathrm{~mL}$ tubes and $15 \mathrm{~mL}$ tubes $\backslash($ BD Falcon 352070; 352097); $1.5 \mathrm{~mL}$ microcentrifuge tubes $\backslash($ Starlab E1415-1500); $40 \mu \mathrm{m}$ cell strainers $\backslash($ BD Falcon 352340). $\cdot$ IV cannulas, 20g: $\backslash($ BD Venflon 391452) $\cdot 25 \mathrm{~g}$ needles, syringes: $1 \mathrm{~mL}, 2.5 \mathrm{~mL}, 10 \mathrm{~mL} \cdot 96$-well plates $\backslash(\mathrm{U}$-bottom) $\cdot$ Bacterial spreaders/ColiRollers plating beads $\backslash$ (Novagen 71013$) \cdot$ Bench-top centrifuge $\cdot$ Microcentrifuge $\cdot$ Liquid nitrogen

\section{Procedure}

**S. pneumoniae stock preparation** 1. Innoculate $10-20 \mathrm{~mL}$ Todd-Hewitt broth $\backslash($ Oxoid) supplemented with $0.5 \%$ yeast extract $\backslash$ (Oxoid) with_S. pneumoniae_ and culture overnight at $37^{\circ} \mathrm{C}, 5 \% \mathrm{CO}_{2} .2$.

Subculture into fresh pre-warmed Todd-Hewitt broth with yeast extract at a ratio of 1:50 and grow to midlog phase $\backslash\left(\mathrm{OD}_{600}=0.5-0.7\right)$ at $37^{\circ} \mathrm{C}, 5 \% \mathrm{CO}_{2}$. 3. Collect the bacteria by centrifugation at $3000 \mathrm{xg}$ for $20 \mathrm{~min}$ at $4^{\circ} \mathrm{C}$ and resuspended at $\mathrm{OD}_{600}=1.0$ in cold PBS $/ 20 \%$ glycerol $\backslash($ Sigma-Aldrich), keep on ice while preparing $1 \mathrm{~mL}$ aliquots. 4. Proceed to snap freeze aliquots in liquid $\mathrm{N}_{2} \backslash$ (keep a non-frozen control aliquot on ice to assess viability). 5 . Assess stocks for viable CFU counts and homogeneity by plating out serial dilutions of three frozen samples and 1 non-frozen control sample on blood agar plates $\backslash$ (LB agar, supplemented with $5 \%$ defibrinated sheep blood $\backslash\left(\right.$ Oxoid)) 6 . Incubate the plates for $24 \mathrm{~h}$ at $37^{\circ} \mathrm{C}, 5 \% \mathrm{CO}_{2}$. 7. Confirm_S. pneumoniae_ colonies by the presence of an a-haemolytic zone and sensitivity to optochin $\backslash$ (Sigma-Aldrich). 8. Count colonies and calculate stock CFU concentration. \(Viability of frozen stocks should be $>90 \%$ compared to the non-frozen control sample). 9 . Maintain virulent stocks by performing _in vivo_ passage every 6-12 months $\backslash$ (Infect a mouse with_S. pneumoniae_, collect the spleen $24 \mathrm{~h}$ post infection. Culture_S. pneumoniae_ colonies from the spleen and use collected colonies to inoculate broth in step 1). ${ }^{*}$ Intranasal administration of S. pneumoniae ${ }^{\star \star} \_$Note 1: There is a considerable difference in virulence between S. pneumoniae strains and serotypes, and also variation within the same strain and serotype between laboratories. Therefore, it is important to perform dose-finding pilot experiments before embarking on a full-scale study.__Note 2: Full training and competency assessment in anaesthesia of laboratory animals is required._ 1 . Thaw frozen stocks and wash twice by centrifugation $\backslash(5000 \mathrm{xg}$ for 5min) in sterile PBS 2. Resuspend at $4 \times 10^{7} \mathrm{CFU} / \mathrm{mL}$ in PBS 3. Keep the suspension on ice at all times and use for infection within 2 hours of thawing $\backslash$ (no loss of viability was observed under these conditions). 4. Induce anaesthesia in mice by inhalation of $3 \%$ isoflurane for $2-3 \mathrm{~min}$ and maintain with $2 \%$ isoflurane for up to $6 \mathrm{~min}$. 5. Visually, respiration should appear slow, deep and even \(not gasping), with no reflex present if the nose is gently touched with clean tissue paper. 6 . Hold the mouse in a supine position, allowing the head to tilt slightly backwards. 7. Mix the_S. pneumoniae_suspension well and collect $50 \mu \mathrm{L}$ $\backslash$ (containing $2 \times 10^{6} \mathrm{CFU}$ ) using a pipette, and place small droplets of the suspension on the nostrils, allowing the mouse to inhale each droplet before placing the next one. 8 . Once the full $50 \mu \mathrm{L}$ dose is 
inhaled, place the mouse in the cage in a supine position and observe to confirm inhalation of the dose and full recovery from anaesthesia: mice should re-gain their righting reflex within 1 minute and normal mobility within 3 minutes. 9 . After infecting all the mice in the study group, prepare serial dilutions of the inoculum and plate on blood agar plates to confirm the infection dose. ${ }^{\star}$ S. pneumoniae survival studies: ${ }^{\star *}$ 1. Record pre-infection body weights and assess animals to ensure they are in good health. 2 . Infect mice intranasally with_S. pneumoniae_as described above. 3. Record body weights daily $\backslash$ (preferably at the same time every day), starting at $24 \mathrm{~h}$ post infection 4 . Monitor animals at least three times a day for a period of 10 days post infection: When signs of sickness are observed monitoring frequency should be increased, including out of hours checks. 5 . We assess disease progression by assigning clinical scores according to the scheme below $\backslash$ (Table 3). Animals are culled when they show $>25 \%$ bodyweight loss or reach score 3 . The most frequently observed clinical signs are piloerection, hunched posture, tremor, and laboured breathing. Rarely, mice may show signs of inner ear infection: head tilting, loss of balance or circling. These animals are culled straight away and excluded from the study. 6. Collect blood and tissues for analysis when animals are culled and/or at the study end-point. See figure in Figures section. ${ }^{*}$ Vaccines and treatments ${ }^{\star *} 1$. To induce a T-independent antibody response, immunise mice with Pneumovax II \(pneumococcal polysaccharide vaccine, Sanofi Pasteur MSD); To induce a T-dependent antibody response, use Prevenar $13 \backslash$ (pneumococcal 13-valent conjugate vaccine, Diphtheria CRM197 protein, Pfizer). a) Pneumovax: Immunise mice with one $100 \mu \mathrm{L} \backslash(0.4 \mu \mathrm{g})$ dose $\backslash(1: 12.5$ dilution in sterile PBS) by intraperitoneal injection, 14 days before infection with _S. pneumoniae_. Include a PBS treated vehicle control group. b) Prevenar: Immunise mice with four 100 $\mu \mathrm{L} I$ $(0.34 \mu \mathrm{g})$ doses $\backslash(1: 6.4$ dilution in sterile PBS) given 14 days apart by intraperitoneal injection, with the last dose 14 days before infection with_S. pneumoniae_. Include a PBS treated vehicle control group. 2. Collect serum from pre- and post-immunisation blood samples to assess antibody levels. 3. \(Optional) Other treatments can be applied before or after_S. pneumoniae_ infection to assess their effects on disease progression and/or immune response. Some examples include cytokines, neutralising antibodies and small molecule inhibitors. ${ }^{*}$ Single cell isolation: Lung and spleen ${ }^{\star *} 1$. Apply treatments and/or infect mice with_S. pneumoniae_as described above. 2. At the desired timepoint post-infection, euthanise mice by $\mathrm{CO}_{2}$ inhalation followed by gentle cervical dislocation to confirm death $\backslash$ (Avoid damage to cervical blood vessels and trachea). 3. Collect blood samples from the abdominal aorta into appropriate containers: Serum collection tubes $\backslash$ (BD microtainer, SST) or $\mathrm{K}^{+}$EDTA coated tubes $\backslash$ (Sarstedt Microvette) for plasma and/or blood cell analysis. 4. \(optional) Collect bronchial-alveolar lavage \(BAL) fluid: a. Use a needle to create a small opening in the trachea above the larynx b. Using a syringe and $20 \mathrm{~g}$ IV cannula \(BD Venflon 391452) \(needle removed), gently inflate the lungs with $1 \mathrm{~mL}$ cold PBS, and withdraw the liquid from the lungs. Repeat three times. c. Place BAL fluid in a microcentrifuge tube on ice 5. Perfuse the lungs with $10 \mathrm{~mL}$ cold PBS through the right ventricle. a. Make a small cut in the left atrium b. Gently hold the heart with forceps, insert a $25 \mathrm{~g}$ needle into the right ventricle and gently flush through $10 \mathrm{~mL}$ cold PBS until the lungs appear white. _Note: in the presence of infection dark patches may remain._ 6. Collect lungs and spleens into cold PBS and keep on ice. \(Weigh tissues for accurate CFU and cell count calculation) 7. Dissociate lungs using the Miltenyi lung dissociation kit and GentleMACS tissue dissociator: a. Place lungs into Miltenyi C-tubes with $2.5 \mathrm{~mL}$ dissociation buffer containing 
enzymes $A$ and $D \backslash($ Miltenyi lung dissociation kit 130-095-927). _Note: for manual dissociation, place lungs in $35 \mathrm{~mm}$ petri dishes with $0.5 \mathrm{~mL}$ cold PBS_b. Homogenise tissue using the Miltenyi GentleMACS $\backslash$ (lung programme 2) and then incubate with the dissociation buffer at $37^{\circ} \mathrm{C}$ for $45 \mathrm{~min}$ with intermittent mixing. _Note: for manual dissociation: chop lung samples into $<1 \mathrm{~mm}$ pieces using a blade, then transfer to $15 \mathrm{~mL}$ tubes containing Miltenyi lung dissociation kit enzyme mixture for incubation._ c. Place the samples on ice and homogenise a second time using the Miltenyi GentleMACS \(lung programme 2). _Note: for manual dissociation, push homogenate through a $100 \mu \mathrm{m}$ cell strainer using a $2.5 \mathrm{~mL}$ syringe plunge and rinse through with 2-3 mL PBS._ d. Transfer the homogenate to $15 \mathrm{~mL}$ tubes $\backslash$ (BD Falcon) and wash by centrifugation $\backslash(500 \mathrm{xg})$ in $10 \mathrm{~mL}$ cold PBS. e. Resuspend the pellet in $3 \mathrm{~mL} 37.5 \%$ isotonic Percoll $\backslash$ (Sigma) at room temperature and centrifuge at $650 \mathrm{xg}$ for $20 \mathrm{~min}$ with low acceleration and no brake. $\mathrm{f}$. Carefully remove the supernatant containing debris using a pipette, wash the cell pellet in PBS and resuspended in 200-500 $\mu \mathrm{L}$ cold PBS. _Note: Where lung CFU counts are also required, process the right lung as described above, and the left lung as described below $\backslash$ (S. pneumoniae CFU counts)._ 8 . Homogenise spleens by pushing through $40 \mu \mathrm{m}$ cell strainers $\backslash(\mathrm{BD})$ a. use a $2.5 \mathrm{ml}$ syringe plunge and rinse the strainers in $2 \mathrm{ml}$ PBS. _Note: Where CFU counts are required, transfer a $200 \mu \mathrm{L}$ sample to a sterile U-bottom 96-well plate and keep on ice before proceeding to $\backslash(\mathrm{b})$._ b. Transfer the cell suspension to a $15 \mathrm{ml}$ Tube $\backslash(\mathrm{BD}$ falcon) and wash once by centrifugation in $5 \mathrm{ml}$ cold PBS. c. Resuspend cell pellets in red blood cell lysis buffer $\backslash$ (Sigma) $\backslash(2 \mathrm{~mL}$ per spleen) and incubate for $5 \mathrm{~min}$ at room temperature. d. Quench lysis by adding $10 \mathrm{ml}$ cold PBS and collect the cells by centrifugation. e. Resuspend the cell pellets in 2$5 \mathrm{ml}$ cold PBS. 9. Collect cells from BAL fluid by centrifugation at $500 \mathrm{xg} \backslash$ (BAL supernatant can be stored at $-20^{\circ} \mathrm{C}$ for cytokine/protein analysis). Resuspend cells in $200-500 \mu \mathrm{L}$ PBS and keep on ice. ${ }^{*} \mathrm{~S}$. pneumoniae CFU counts ${ }^{\star \star} 1$. Place the lungs/left lung in a $2 \mathrm{~mL}$ Eppendorf $\backslash($ Safelock) microfuge tube/ screw cap tube containing $1 \mathrm{~mL}$ PBS and $3 \times 3.2 \mathrm{~mm}$ stainless steel beads $\backslash($ Next Advance). 2. Place the tubes in a Next Advance Bullet Blender and homogenise at speed 8 for $3 \mathrm{~min}$. 3. Transfer $200 \mu \mathrm{L}$ of the homogenate into a U-bottom 96 well plate and keep on ice. 4. Perform 10-fold serial dilutions for spleen $\backslash$ (see Single cell isolation: Lung and spleen step 8a) and lung homogenates 5. Plate out $100 \mu \mathrm{L}$ samples using ColiRollers $\backslash$ (Novagen) or a bacterial spreader on blood agar plates and incubate $24 \mathrm{~h}$ at $37^{\circ} \mathrm{C} .6$. Count_S. pneumoniae_ colonies on plates with 10-300 colonies. \(recognised by the presence of a green a-haemolytic zone). ${ }^{* *}$ Immune cell characterisation by flow cytometry** 1 . Transfer $200 \mu \mathrm{L}$ samples of spleen and lung single cell suspensions to U-bottom 96 well plates. 2. Prepare surface antibody master mix containing FC block and viability dye in staining buffer $\backslash(0.5 \%$ BSA in PBS) 3 . Collect samples by centrifugation and resuspend in $50 \mu \mathrm{L}$ antibody master mix per sample. 4. Protect plates from light and incubate at $4^{\circ} \mathrm{C}$ for $30-40 \mathrm{~min}$. 5. Quench staining by adding $150 \mu \mathrm{L}$ staining buffer. 6 . Collect cells by centrifugation and wash once with $200 \mu \mathrm{L}$ staining buffer. 7 . Fix samples in $50 \mu \mathrm{L} 4 \%$ paraformaldehyde in PBS $\backslash$ (Biolegend) for $10 \mathrm{~min}$ at room temperature. _Note: where intracellular staining is required $\backslash$ (e.g. transcription factors), use the appropriate fixation buffer from Transcription factor staining buffer set $\backslash$ (eBioscience) or alternative._ 8. Quench fixation by adding $150 \mu \mathrm{L}$ staining buffer 9 . Collect cells by centrifugation and wash twice with $200 \mu \mathrm{L}$ staining buffer. 10 . Where no intracellular staining is required, resuspend samples in 50-100 $\mu \mathrm{L}$ PBS, add non fluorescent counting beads, and analyse on a flow cytometer. $\backslash$ (Alternatively, fixed samples can be stored at $4^{\circ} \mathrm{C}$ up to $48 \mathrm{~h}$ before analysis) _Note: Where 
intracellular staining is required collect the cells by centrifugation and wash once with an appropriate permeabilisation buffer $\backslash$ (e.g. Transcription factor staining buffer set, eBioscience). Resuspend cells in $50 \mu \mathrm{L}$ intracellular antibody master mix in permeabilisation buffer. Protect samples from light and incubate at room temperature for $40 \mathrm{~min}$. Quench staining, wash, resuspend and analyse cells as described in steps 8-10._ 11. Before analysing samples by flow cytometry, ensure that the appropriate unstained and single stained compensation controls as well as the required fluorescence-minus-one $\backslash$ (FMO) or isotype staining controls are prepared.

\section{Timing}

**S. pneumoniae stock preparation:** 2 days **Intranasal administration of S. pneumoniae** $1.5-2 \mathrm{~h}$ for a group of 20 mice $* * S$. pneumoniae survival studies:** 10 days **Vaccines ${ }^{\star *}$ Injections: $1-2 \mathrm{~h}$ for a group of 20 mice Pneumovax II vaccination schedule: 14 days Prevenar-13 vaccination schedule: 8 weeks **Single cell isolation: Lung and spleen** Dissections and cell isolation for a group of 20 mice: 10-12h $\backslash$ (13-15h $+24 \mathrm{~h}$ incubation to include CFU counts) $* \star S$. pneumoniae CFU counts** Dissections, tissue processing and plating for a group of 20 mice: $5-6 \mathrm{~h}+24 \mathrm{~h}$ incubation $* *$ Immune cell characterisation by flow cytometry* Surface stain only: 2-3 hours Surface and intracellular stain: 3-4 hours Flow cytometry analysis time depends on the instrument used as well as the number of samples and panels to be analysed.

\section{Troubleshooting}

$\star \star$ S. pneumoniae stock preparation ${ }^{\star \star * *}$ Contamination:** • As no antibiotics are used, cultures can easily become contaminated. Use a high-level disinfectant such as Distel, Trigene or Virkon to clean all work surfaces, and ensure sterility of flasks and growth medium. ${ }^{*}$ Poor viability/growth:** • Viability will decrease when cultures are allowed to grow past mid-log phase. Take regular $\mathrm{OD}_{600}$ readings and proceed to collect bacteria using a pre-cooled centrifuge as soon as the culture reaches $\mathrm{OD}_{600}$ 0.5-0.7. Prolonged periods at room temperature could also affect viability, therefore work quickly to snap-freeze aliquots as soon as possible after the culture is harvested and keep samples on ice at all times during the process. Poor growth on blood ager plates/ absence of a clear a-haemolytic ring around_S. pneumoniae_ colonies can result from lysis of the sheep blood. Ensure the agar cools to $50^{\circ} \mathrm{C}$ before adding the blood as higher temperatures will result in lysis. Store prepared plates at $4^{\circ} \mathrm{C}$ for up to 5 days. Blood agar plates should appear opaque and will become transparent when lysis occurs. $* \star$ Intranasal administration of S. pneumoniae and survival studies ${ }^{\star \star *}$ Animals appear unwell within minutes/hours after S. pneumoniae administration:** • Contaminating species and/or toxins released as a result of bacterial death and lysis can cause animals to become unwell soon after intranasal administration. Ensure_S. pneumoniae_stocks are pure with high viability before administration to animals. Keep diluted _S. pneumoniae_stock on ice at all times and administer to animals within two hours of defrosting a frozen aliquot. ${ }^{*}$ Animals recover from anaesthesia before inhaling the full dose:** Anaesthesia is not deep enough: Ensure animals are breathing deeply and evenly and show no reflex when the nose is 
touched with a piece of clean tissue paper. The entire $50 \mu \mathrm{L}$ dose should be administered within $20-30 \mathrm{~s}$, allowing roughly $1 \mathrm{~min}$ for the animal to remain in a supine position before recovery. ${ }^{\star \star}$ Animals take longer than expected to recover from anaesthesia/gasping breathing is present: $* *$ Anaesthesia is too deep. Remove animals from the anaesthetic chamber and observe to ensure full recovery. Animals should be closely observed while in the anaesthetic chamber at all times. ${ }^{*}$ Higher/lower than expected mortality rates following S. pneumoniae infection: $* \star$ S Since there is considerable variation in virulence between different_S. pneumoniae_strains and serotypes, pilot studies should be used to determine the optimum dose. - Repeated_in vitro_ passage of _S. pneumoniae_stock can lead to reduced virulence over time. Therefore, it is recommended to perform _in vivo_ passage by culturing new stock from _S. pneumoniae_ colonies recovered from the spleens of infected mice every 6-12 months. P Plating out the diluted_S. pneumoniae_stock used for infection is recommended to confirm viability and absence of contamination, as it is often helpful to rule out these factors should the study not progress as expected. $\star *$ Immune cell characterisation by flow cytometry* $\cdot$ Multicolour antibody panels require optimisation and small pilot experiments are recommended to establish a panel for larger studies. High autofluorescence is a characteristic of several cell types found in the lung, most notably alveolar macrophages. It is therefore recommended to avoid fluorochromes with low peak emission wavelengths for identification of alveolar macrophages $\backslash(P E$ and Alexa-647 are good choices for these cells). In addition, fluorescence-minus-one controls should be used to set gates accurately. $\cdot$ High background and non-specific staining can also result from high levels of debris/dead cells in the samples and could be a problem especially in lung samples. High levels of debris/clumps of dead cells can also block the flow cytometer and lead to aberrant results. The Percoll separation step should remove debris but if some remain the samples can be filtered prior to analysis. CellTrics $30 \mu \mathrm{m}$ filters $\backslash($ SYSMEX) work well for small sample volumes. Always use a viability dye to exclude dead cells.

\section{Anticipated Results}

**S. pneumoniae stock preparation** Frozen_S. pneumoniae_stocks should be free of contamination and have $>90 \%$ viability compared to a non-frozen control aliquot. To avoid adverse effects, stocks with $<90 \%$ viability should not be used for administration to animals. In addition, purity should be confirmed in undiluted stock samples by observing a clear optochin inhibition ring on blood agar plates, as well as a green, translucent a-haemolytic zone around individual colonies in diluted samples. ${ }^{\star *}$ Intranasal administration of S. pneumoniae, survival studies and vaccines** After intranasal administration of _S. pneumoniae_, mice are expected to recover from anaesthesia within 3 minutes, and the mice are expected to appear well with normal levels of activity and no respiratory abnormalities. In our hands, one dose of $2 \times 10^{6}$ CFU_S. pneumoniae_TIGR4, serotype 4 results in 30-50\% of wildtype C57BI/6 mice surviving to 10 days post infection. At 6-8 hours post infection the mice may start to show transiently reduced activity levels and appear subdued; these effects are expected to disappear by $24 \mathrm{~h}$ post infection. However, at $24 \mathrm{~h}$ post infection mice are expected to start showing signs of weight loss, followed by increased weight loss and signs of sickness as described in Table 3 from 36-48h onwards. Animals that do not reach the humane end-point during this time are expected to start recovering from 96 hours onwards, and all 
surviving animals are expected to reach their pre-infection weight by 10 days post infection. It is important to ensure animals in experimental and control groups are age and sex matched [9]. We found that males are more susceptible to _S. pneumoniae_ infection compared to female mice, although this was not associated with increased weight loss in male mice \(Figure 1). Immunisation with Pneumovax or Prevenar-13 prior to infection is expected to provide $>90 \%$ protection against disease progression in wildtype C57BI/ 6 mice $\backslash\left(\right.$ Figure 2). See figure in Figures section. See figure in Figures section. ${ }^{*} \mathrm{CFU}$ counts, Single cell isolation and Immune cell characterisation** We focused our CFU count and immune cell characterisation studies on the $24 \mathrm{~h}$ post infection time-point because we are interested in the immune response to acute infection. At 24h most animals start to show weight loss but appear clinically healthy. These analyses can also be applied to other time-points; However, it should be considered that the interpretation of results at later time-points can be complicated because study groups will contain individuals with a wide variation in health status which could affect experimental readouts independently of genotype or treatment. In addition, analysis at later time-points will invariably select for individuals surviving to that timepoint. We expect lung CFU counts to remain stable for the first 6 hours post infection, then reduce and become more variable from $24 \mathrm{~h}$ onwards. We expect mice to start showing bacteraemia as measured by CFU in the spleen from 24h onwards $\backslash($ Figure 3 ). See figure in Figures section. The suggested flow cytometry staining panels allows the detection of different lymphocyte and myeloid populations of interest to our studies and should be adjusted to individual research questions. A detailed flow cytometry protocol for the identification of lung macrophage and dendritic cell subsets is provided by Misharin et al $\backslash[10]$. Examples of gating strategies for the identification of lymphocyte and myeloid cell populations in the lung at 24h post_S. pneumoniae_ infection are shown in Figures 4 and 5 respectively. See figure in Figures section. See figure in Figures section. We do not observe significant lymphocyte infiltration in the lungs during the first $24 \mathrm{~h}$ post infection. Alveolar macrophage numbers are also expected to remain constant during the first $24 \mathrm{~h}$ post infection, while neutrophils and inflammatory monocytes are expected to increase from 6-8h onwards, peak at $24 \mathrm{~h}$ post infection and then start to decrease $\backslash$ (Figure 6). See figure in Figures section.

\section{References}

1. Weiser, J.N., D.M. Ferreira, and J.C. Paton, Streptococcus pneumoniae: transmission, colonization and invasion. Nat Rev Microbiol, 2018. 16\(6): p. 355-367. 2. Morris, D.E., D.W. Cleary, and S.C. Clarke, Secondary Bacterial Infections Associated with Influenza Pandemics. Front Microbiol, 2017. 8: p. 1041. 3. Halpin, D.M., et al., Impact and prevention of severe exacerbations of COPD: a review of the evidence. Int J Chron Obstruct Pulmon Dis, 2017. 12: p. 2891-2908. 4. Daniels, C.C., P.D. Rogers, and C.M. Shelton, A Review of Pneumococcal Vaccines: Current Polysaccharide Vaccine Recommendations and Future Protein Antigens. J Pediatr Pharmacol Ther, 2016. 21\(1): p. 27-35. 5. Chiavolini, D., G. Pozzi, and S. Ricci, Animal models of Streptococcus pneumoniae disease. Clin Microbiol Rev, 2008. 21\(4): p. 666-85. 6. Stark, A.K., et al., PI3Kdelta hyper-activation promotes development of B cells that exacerbate Streptococcus pneumoniae infection in an antibody-independent manner. Nat Commun, 2018. 9\(1): p.

3174. 7. FELASA recommendations for the health monitoring of mouse, rat, hamster, guinea pig and 
rabbit colonies in breeding and experimental units. Lab Anim, 2014. 48\(3): p. 178-192. 8. Sandgren, A., et al., Virulence in mice of pneumococcal clonal types with known invasive disease potential in humans. $J$ Infect Dis, 2005. 192\(5): p. 791-800. 9. Kadioglu, A., et al., Sex-based differences in susceptibility to respiratory and systemic pneumococcal disease in mice. J Infect Dis, 2011. 204\(12): p. 1971-9. 10. Misharin, A.V., et al., Flow cytometric analysis of macrophages and dendritic cell subsets in the mouse lung. Am J Respir Cell Mol Biol, 2013. 49\(4): p. 503-10.

\section{Acknowledgements}

We are grateful for the support and expert advice from the Babraham Institute Biological Services Unit and Flow Cytometry Facilities.

\section{Figures}
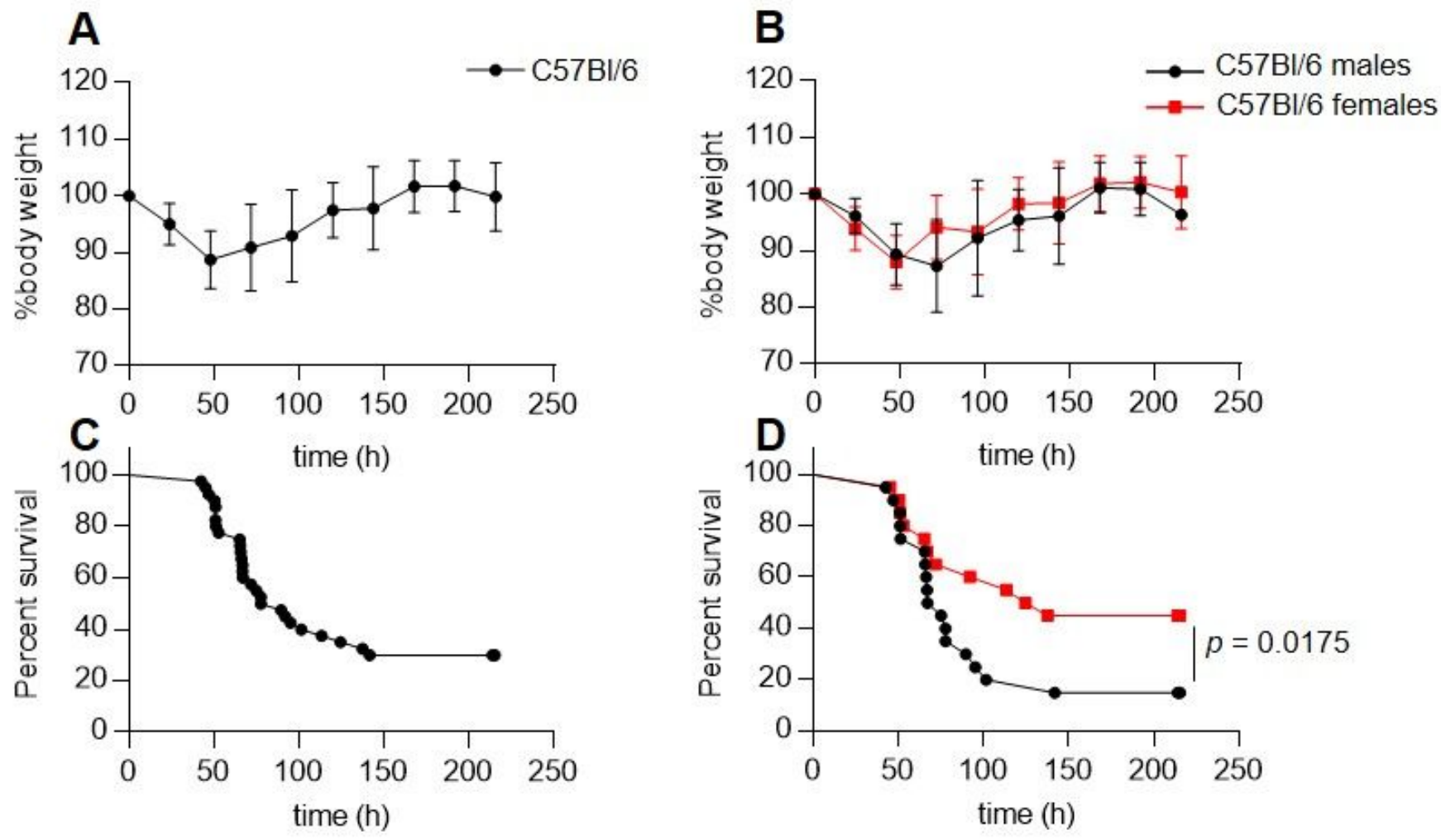

Figure 1: Expected disease progression following intranasal S. pneumoniae TIGR4, serotype 4 infection. $\mathrm{C} 57 \mathrm{BI} / 6$ mice were infected intranasally with 1 dose of $2 \times 10^{6} \mathrm{CFU} S$. pneumoniae TIGR4, serotype 4 in $50 \mu \mathrm{L}$ PBS. Weight loss and disease progression was monitored over 10 days. A: Expected weight loss for a combined group of $50 \%$ males and $50 \%$ females. B: Expected weight loss is similar for males and females. C: Expected mortality for a combined group of $50 \%$ males and $50 \%$ females. D: C57BI/6 males show significantly lower survival rates in response to $S$. pneumoniae infection compared to females.

Combined C57BI/6 wildtype control groups from 2 independent experiments $(n=40)$.

\section{Figure 1}


*Expected disease progression following intranasal_S. pneumoniae__TIGR4, serotype 4 infection* C57BI/ 6 mice were infected intranasally with 1 dose of $2 \times 10^{\wedge} 6^{\wedge}$ CFU_S. pneumoniae_TIGR4, serotype 4 in $50 \mu \mathrm{L}$ PBS. Weight loss and disease progression was monitored over 10 days. *A:* Expected weight loss for a combined group of $50 \%$ males and $50 \%$ females. ${ }^{*} \mathrm{~B}:{ }^{*}$ Expected weight loss is similar for males and females. ${ }^{\star} \mathrm{C}$ :* Expected mortality for a combined group of $50 \%$ males and $50 \%$ females. ${ }^{*} \mathrm{D}:{ }^{*} \mathrm{C} 57 \mathrm{BI} / 6$ males show significantly lower survival rates in response to _S. pneumoniae_ infection compared to females. Combined C57BI/ 6 wildtype control groups from 2 independent experiments $(n=40)$.
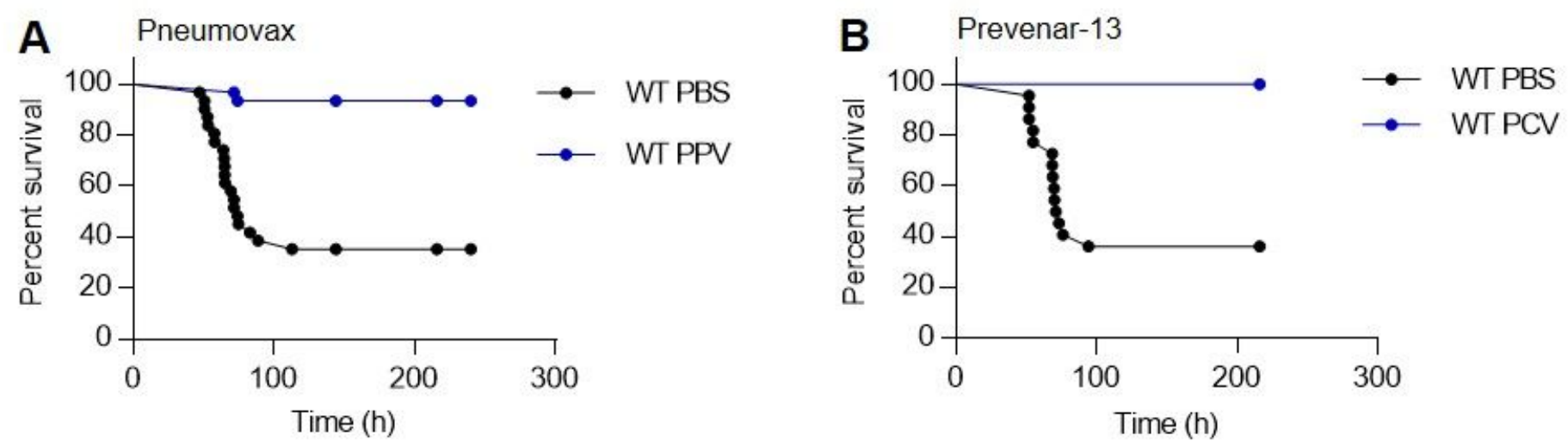

Figure 2: Pneumovax and Prevenar-13 treatment protects against S. pneumoniae TIGR4, serotype 4 infection. C57BI/6 mice were treated with Pneumovax or Prevenar-13 together with an age and sex matched PBS vehicle control group prior to intranasal infection with $2 \times 10^{6} \mathrm{CFU}$ S. pneumoniae TIGR4, serotype 4 in $50 \mu \mathrm{L}$ PBS. A: Pneumovax treatment resulted in $93 \%$ survival compared to $35 \%$ survival in the vehicle treated group. B: Prevenar-13 treatment resulted in $100 \%$ survival compared to $36 \%$ survival in the vehicle control treated group.

A: Combined Pneumovax or Prevenar-13 and PBS vehicle treated C57BI/6 wildtype control groups from 3 independent experiments $(n=31)$.

\section{Figure 2}

*Pneumovax and Prevenar-13 treatment protects against_S. pneumoniae_TIGR4, serotype 4 infection* C57BI/ 6 mice were treated with Pneumovax or Prevenar-13 together with an age and sex matched PBS vehicle control group prior to intranasal infection with $2 \times 10^{\wedge} 6^{\wedge}$ CFU_S. pneumoniae_ TIGR4, serotype 4 in $50 \mu \mathrm{L}$ PBS. *A:* Pneumovax treatment resulted in $93 \%$ survival compared to $35 \%$ survival in the vehicle treated group. *B:* Prevenar-13 treatment resulted in $100 \%$ survival compared to $36 \%$ survival in the vehicle control treated group. Combined Pneumovax or Prevenar and PBS vehicle treated C57BI/6 wildtype control groups from 3 independent experiments $(n=31)$. 

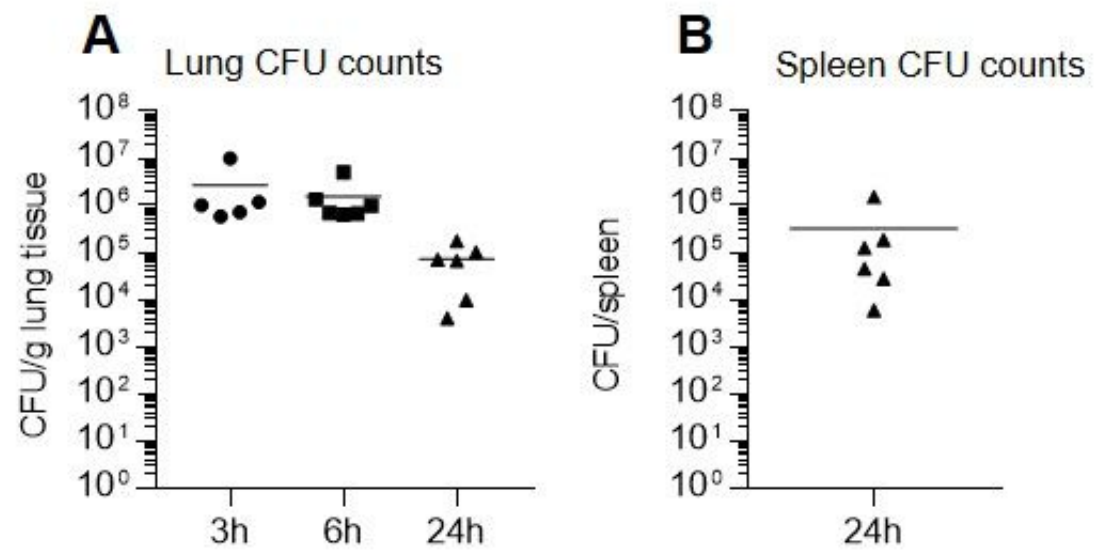

Figure 3: Expected CFU counts at 24h post S. pneumoniae TIGR4, serotype 4 infection.

C57BI/6 mice were intranasally infected with $2 \times 10^{6} \mathrm{CFU} S$. pneumoniae TIGR4, serotype 4 in $50 \mu \mathrm{L}$ PBS. A: In wildtype mice, lung CFU counts are expected to remain stable for the first 6 hours post infection, then become lower and more variable from $24 \mathrm{~h}$ onwards. B: Mice are expected to become bacteraemic from $24 \mathrm{~h}$ onwards.

C57BI/6 wildtype control groups from 1 representative experiment $(n=6)$.

\section{Figure 3}

*Expected CFU counts at 24h post_S. pneumoniae__TIGR4, serotype 4 infection. * C57BI/6 mice were intranasally infected with $2 \times 10^{\wedge} 6^{\wedge} \mathrm{CFU} \_S$. pneumoniae_TIGR4, serotype 4 in $50 \mu \mathrm{L}$ PBS. *A:* In wildtype mice, lung CFU counts are expected to remain stable for the first 6 hours post infection, then become lower and more variable from $24 \mathrm{~h}$ onwards. ${ }^{*} \mathrm{~B}$ ** Mice are expected to become bacteraemic from $24 \mathrm{~h}$ onwards. C57BI/6 wildtype control groups from 1 representative experiment $(n=6)$. 

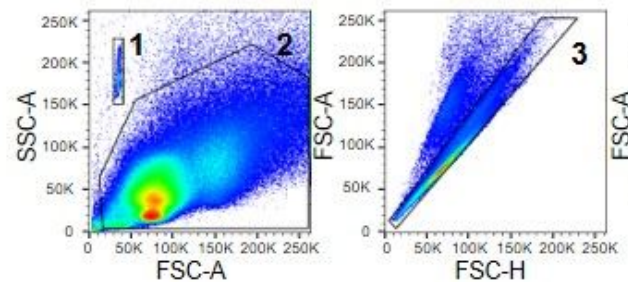

FSC-H

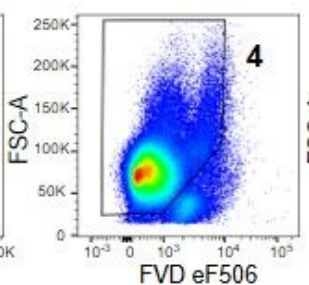

FVD eF506

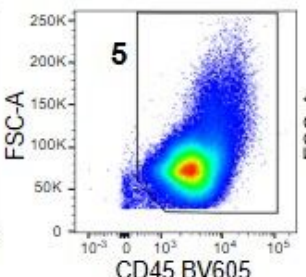

CD45 BV605
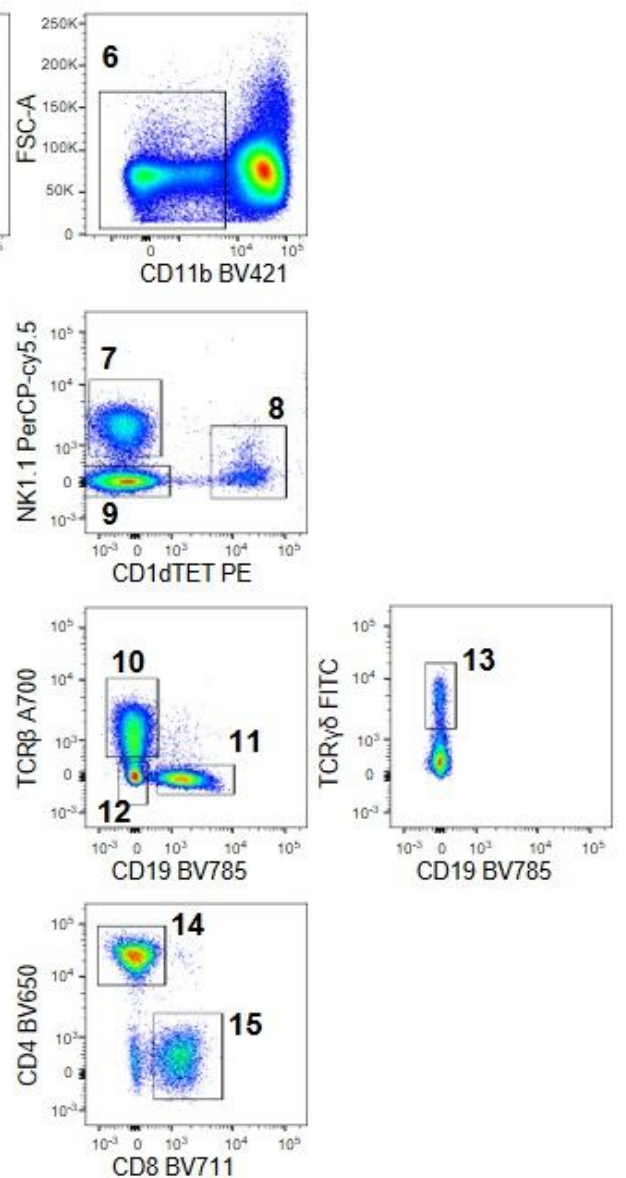

Gate on counting beads

Gate on total cell population identified by forward and side scatter.

Gate on single cells identified by forward scatter peak height and width

4. Gate on live cells: identified by low amine reactive fixable viability dye (FVD) staining

5. Gate on $\mathrm{CD}_{4} 5^{+}$Leucocytes

6. Exclude neutrophils and other myeloid cells by gating on $\mathrm{CD} 11 \mathrm{~b} / \mathrm{CD} 11 \mathrm{~b} \mathrm{~b}^{\circ}$ cells

7. Identify NK cells by NK1.1 expression

8. Identify NKT cells by CD1d-tetramer staining

9. Gate on NK1.1, CD1d-tetramer cells

10. Identify conventional $\alpha \beta T$ cells by TCR $\beta$ expression

Gate on TCR $\beta^{+}$cells then continue to step 14 and 15

11. Identify $\mathrm{B}$ cells by $\mathrm{CD} 19$ expression

12. Gate on TCR $\beta$; CD19- cells

13. Identify Yठ̄T cells by TCRYō expression

14. Identify $\mathrm{CD}^{+}{ }^{+} \mathrm{T}$ cells by $\mathrm{CD} 4$ expression

15. Identify $\mathrm{CD}^{+} \mathrm{T}$ cells by $\mathrm{CD} 8$ expression

Figure 4: Example Gating strategy for the identification of lung lymphocyte populations

C57BI/ 6 mice were infected intranasally with 1 dose of $2 \times 10^{6} \mathrm{CFU}$ S. pneumoniae TIGR4, serotype 4 in $50 \mu \mathrm{L}$ PBS. Lungs were collected $24 \mathrm{~h}$ post infection and single cell suspensions were analysed by flow cytometry

\section{Figure 4}

*Example Gating strategy for the identification of lung lymphocyte populations* C57BI/6 mice were

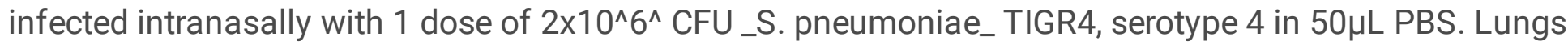
were collected $24 \mathrm{~h}$ post infection and single cell suspensions were analysed by flow cytometry 

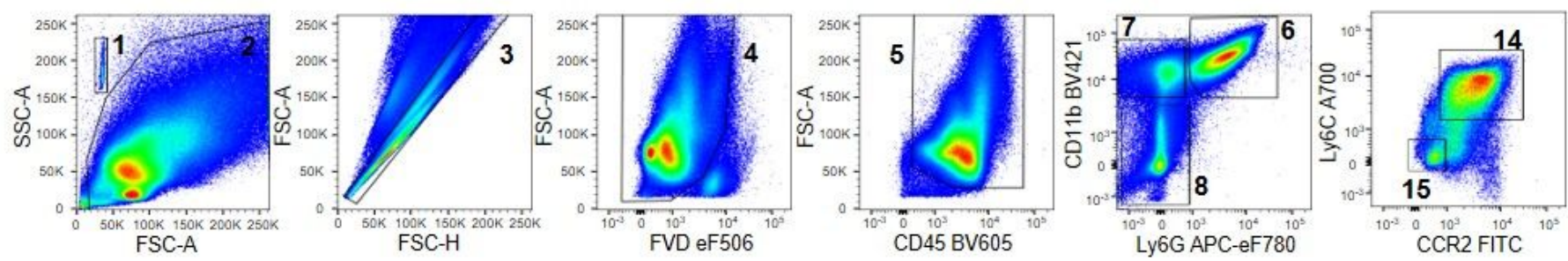

1. Gate on counting beads

2. Gate on total cell population identified by forward and side scatter.

3. Gate on single cells identified by forward scatter peak height and width

4. Gate on live cells: identified by low amine reactive fixable viability dye (FVD) staining

5. Gate on $\mathrm{CD}_{4} 5^{+}$Leucocytes

6. Identify neutrophils by high CD11b and Ly6G expression

7. Gate on CD11 bigh Ly6G- cells, continue to step 14, 15

8. Gate on CD11 b-/lo Ly6G- cells

9. Identify alveolar macrophages by high CD11c and SiglecF expression

10. Gate on CD11 $\mathrm{c}^{-/ / 0}$ SiglecF- cells

11. Exclude conventional T cells, B cells and NK cells by gating on CD3-CD19-NK1.1- cells

12. Gate on Ly6C+ ${ }^{+}$cells: $\mathrm{CD} 11 \mathrm{~b}^{\circ} \mathrm{CD} 11 \mathrm{c}^{\mathrm{lo}} \mathrm{Ly} 6 \mathrm{C}^{+} \mathrm{MHCII}$

13. Gate on $\mathrm{MHCll}^{+}$cells: CD $11 \mathrm{~b}^{10} \mathrm{CD} 11 \mathrm{c}^{\mathrm{lo}} \mathrm{Ly} 6 \mathrm{C}-\mathrm{MHCl}{ }^{+}$

14. Identify CD11 bigh $L y 6 C^{+} C C R 2+$ inflammatory monocytes

15. Gate on CD11 bigh Ly6C-CCR2- cells

16. Gate on MHCll- cells: CD11 $\mathrm{b}^{\text {high }} \mathrm{Ly} 6 \mathrm{C}-\mathrm{CCR} 2-\mathrm{MHCll}$ - cells

17. Gate on $\mathrm{MHCII}^{+}$cells: CD11 $\mathrm{b}^{\text {high } L y 6 C-C C R 2-M H C I{ }^{+}}$cells
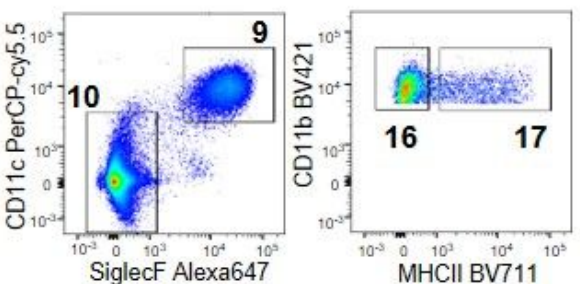

Figure 5: Example gating strategy for the identification of lung myeloid populations

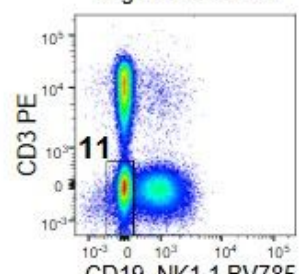

MHCII BV711

$\mathrm{C} 57 \mathrm{BI} / 6$ mice were infected intranasally with 1 dose of $2 \times 10^{6} \mathrm{CFU}$ S. pneumoniae TIGR4, serotype 4 in $50 \mu \mathrm{L}$ PBS. Lungs were collected $24 \mathrm{~h}$ post infection and single cell suspensions were analysed by flow cytometry.

\section{Figure 5}

*Example gating strategy for the identification of lung myeloid populations* $\mathrm{C} 57 \mathrm{BI} / 6$ mice were infected

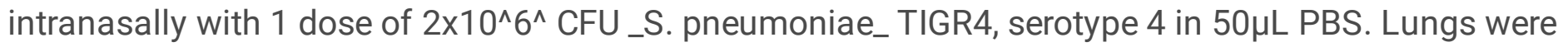
collected $24 \mathrm{~h}$ post infection and single cell suspensions were analysed by flow cytometry. 

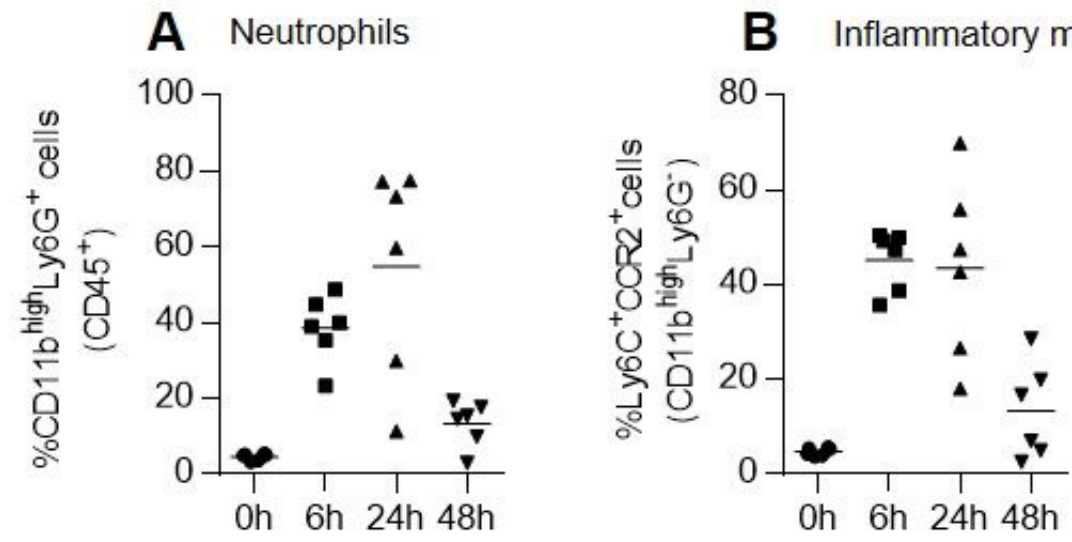

Figure 6: Neutrophil and inflammatory monocyte lung infiltration in response to S. pneumoniae infection. C57BI/6 mice were intranasally infected with $2 \times 10^{6} \mathrm{CFU}$ S. pneumoniae TIGR4, serotype 4 in $50 \mu \mathrm{L}$ PBS. A: In wildtype mice, neutrophil infiltration starts from $3 \mathrm{~h}$ post infection and peak at $24 \mathrm{~h}$ post infection. B: Inflammatory monocyte infiltration starts from $3 \mathrm{~h}$ post infection and remain stable between $6-24 \mathrm{~h}$ post infection. C57BI/6 wildtype control groups from 1 representative experiment $(n=6)$.

\section{Figure 6}

*Neutrophil and inflammatory monocyte lung infiltration in response to _S. pneumoniae_infection.* C57BI/ 6 mice were intranasally infected with $2 \times 10^{\wedge} 6^{\wedge}$ CFU_S. pneumoniae_ TIGR4, serotype 4 in $50 \mu \mathrm{L}$ PBS. *A:* In wildtype mice, neutrophil infiltration starts from $3 \mathrm{~h}$ post infection and peak at $24 \mathrm{~h}$ post infection. * $\mathrm{B}: *$ Inflammatory monocyte infiltration starts from $3 \mathrm{~h}$ post infection and remain stable between $6-24 \mathrm{~h}$ post infection. C57BI/6 wildtype control groups from 1 representative experiment $(n=6)$. 
Table 1: Flow cytometry antibodies

\begin{tabular}{|l|l|l|l|l|}
\hline anti-mouse & Clone & Dilution & Supplier & Cat. no \\
\hline CCR2 & 475301 & $1: 100$ & R\&D Systems & FAB5538A-025 \\
\hline CD11b & M1/70 & $1: 1000$ & Biolegend & 101251 \\
\hline CD11c & N418 & $1: 500$ & Biolegend & 117328 \\
\hline CD19 & $1 \mathrm{D} 3$ & $1: 300$ & Biolegend & 115543 \\
\hline CD1d tetramer (PBS-57) & & $1: 100$ & NIH tetramer core facility & Mouse CD1d PBS-57 \\
\hline CD3 & $145-2 C 11$ & $1: 300$ & Biolegend & 100308 \\
\hline CD4 & RM4-5 & $1: 300$ & Biolegend & 100555 \\
\hline CD45 & $30-F 11$ & $1: 300$ & Biolegend & 103155 \\
\hline CD8a & $53-6.7$ & $1: 300$ & Biolegend & 100759 \\
\hline Ly6C & HK1.4 & $1: 500$ & Biolegend & 128024 \\
\hline Ly6G & 1 A8 & $1: 500$ & Biolegend & 127624 \\
\hline NK1.1 & PK136 & $1: 300$ & Biolegend & 108749,108728 \\
\hline MHCII & M5/114.15.2 & $1: 300$ & Biolegend & 107643 \\
\hline Siglec-F & E50-2440 & $1: 500$ & BD Biosciences & 562680 \\
\hline TCR 3 & H57-597 & $1: 300$ & Biolegend & 109224 \\
\hline p8TCR & UC7-13D5 & $1: 100$ & Biolegend & 107504 \\
\hline Fixable viability dye & & $1: 5000$ & eBioscience & $65-0866-14$ \\
\hline FC Block (CD16/32) & 93 & $1: 1000$ & Biolegend & 101320 \\
\hline
\end{tabular}

Figure 7

Table 1 *Antibodies for flow cytometry* 
Table 2: LSRFortessa configuration

\begin{tabular}{|l|l|l|l|r|}
\hline Laser & Laser power & Make & Filters BP & Filters LP \\
\hline $355 \mathrm{~nm}$ & $20 \mathrm{~mW}$ & Coherent Genesis & $379 / 28$ & \\
\hline & & & $450 / 50$ & 410 \\
\hline $405 \mathrm{~nm}$ & $50 \mathrm{~mW}$ & Coherent OBIS & $450 / 50$ & \\
\hline & & & $525 / 50$ & 505 \\
\hline & & & $610 / 20$ & 600 \\
\hline & & & $660 / 20$ & 630 \\
\hline & & & $710 / 50$ & 690 \\
\hline & & Coherent Sapphire & $530 / 30$ & 750 \\
\hline $488 \mathrm{~nm}$ & $50 \mathrm{~mW}$ & & $710 / 50$ & 505 \\
\hline & & Coherent Sapphire & $585 / 15$ & 685 \\
\hline $561 \mathrm{~nm}$ & $50 \mathrm{~mW}$ & & $610 / 20$ & \\
\hline & & & $670 / 30$ & 600 \\
\hline & & & $710 / 50$ & 635 \\
\hline & & & $780 / 60$ & 685 \\
\hline & & & $670 / 14$ & 750 \\
\hline & & & $730 / 45$ & \\
\hline & & & $780 / 60$ & 690 \\
\hline & & & & 750 \\
\hline & & & & \\
\hline
\end{tabular}

Figure 8

Table 2 *LSRFortessa laser and filter configuration* 
Table 3: Mouse sickness score table

\begin{tabular}{|l|l|l|}
\hline Score & Observations & Action \\
\hline 0 & Healthy & Continue monitoring \\
\hline 1 & $\begin{array}{l}\text { Transiently subdued, reduced response (expected after infection) } \\
\text { Slightly ruffled coat } \\
\text { Transient ocular discharge } \\
\text { Up to 10\% weight loss }\end{array}$ & $\begin{array}{l}\text { Increase monitoring } \\
\text { frequency }\end{array}$ \\
\hline $2-3$ & $\begin{array}{l}\text { Clear piloerection } \\
\text { Intermittent hunched posture } \\
\text { Persistent oculo-nasal discharge (no improvement in 24h) } \\
\text { Persistently subdued, reduced response (no improvement in 24h) } \\
\text { Intermittent abnormal breathing } \\
\text { Up to 15\% weight loss }\end{array}$ & $\begin{array}{l}\text { Animals are culled at this } \\
\text { stage. }\end{array}$ \\
\hline 4 & $\begin{array}{l}\text { Animals found dead/moribund } \\
\text { (a small number of animals (<1\%) may be found dead/moribund } \\
\text { even if they displayed no/mild signs 2-3 hours before) }\end{array}$ & $\begin{array}{l}\text { Animals are culled } \\
\text { immediately }\end{array}$ \\
\hline
\end{tabular}

Figure 9

Table 3 *Mouse sickness scoring table* 